\title{
Research on Power Quality Evaluation Based on Radar Chart Method and Fuzzy Membership Degree
}

\author{
Aiqiang Pan'1, Jian Zhou', Peng Zhang1, Ronghui Liu², Yichao Wang² \\ ${ }^{1}$ Electric Power Research Institute State Grid Shanghai Municipal Electric Power Company, Shanghai, China \\ ${ }^{2}$ College of Electrical Engineering, Shanghai University of Electric Power, Shanghai, China \\ Email: Dsy_panaq@126.com,393684184@qq.com
}

How to cite this paper: Pan, A.Q., Zhou, J., Zhang, P., Liu, R.H. and Wang, Y.C. (2017) Research on Power Quality Evaluation Based on Radar Chart Method and Fuzzy Membership Degree. Energy and Power Engineering, 9, 725-734. https://doi.org/10.4236/epe.2017.94B078

Received: March 10, 2017 Accepted: March 30, 2017 Published: April 6, 2017

\begin{abstract}
Aiming at the current limit value of six steady-state energy indexes, the current radar method is used for reference. A method of comprehensive evaluation of power quality based on improved radar method is proposed, which improves the power quality index Type radar pattern to represent the steady-state indicator. Each of the main indicators corresponds to a partial ring, and the angle of the annular portion is mainly affected by the size of the weight. Compared with the previous radar map method to maintain the independence of the indicators and a single indicator of the binding data assessment. The method has the advantages of good feasibility.
\end{abstract}

\section{Keywords}

Power Quality, Steady-State Index, Sub-Index, Radar Map

\section{Introduction}

Electricity as a commonly used energy, not only economical and easy to control and conversion. The application of energy has become an important indicator of the level of national development. In recent years, with the continuous development of industrialization and national economic level, the whole society is more and more practical to the power, the power quality requirements are gradually improved, the quality of power quality is directly related to the entire power market economic benefits. With the adjustment of energy structure and the rapid development of national economy, long-distance DC transmission and large power grid interconnection to the fundamental changes in the structure of the grid, the resulting AC and DC hybrid operation of the power system and the increasingly variable power grid negative characteristics, As well as the non-li- 
near load and time-varying load in the power grid, brought about such problems as harmonics, voltage fluctuation and flicker, three-phase imbalance [1] [2], which deteriorated the power quality index of the grid. Therefore, it is important to develop a suitable power quality assessment method to evaluate the power quality index synthetically and to improve the power quality to optimize the electricity market.

China has proposed nine national standards for power quality to analyze the power quality problem, which is divided into six steady-state indicators and three transient indicators, because the transient index is not clear data limits are not easy to assess, so the current power quality, the comprehensive evaluation mainly starts from the steady state index. This paper combines the contents of national standard of power quality in China [3] [4] [5] [6], and selects 6 of them as steady-state power quality indicators, which are the allowable deviation of supply voltage, voltage fluctuation, voltage flicker, harmonic distortion rate, three-phase voltage Balance, power system frequency tolerance.

The existing methods of comprehensive evaluation of power quality mainly include three categories [7] [8] [9] based on fuzzy mathematics theory, probability statistics theory and intelligent algorithm. These methods solve the problem of comprehensive evaluation to a certain extent, but the traditional mathematical algorithm is The evaluation of power quality indicators can only stay in the written text, and the radar chart method as the representative of the graphics analysis can be more clearly to show the specific indicators of the specific situation, this paper refers to the radar map in the assessment of indicators Simple and clear, clear the characteristics of the combination of fuzzy membership function to carry out the power quality indicators, so that it can more objectively show the advantages and disadvantages of the indicators.

\section{Indicator Normalization and Selection}

The primary objective of the evaluation of the power quality indicators is to select the appropriate indicators. First, the indicators should be normalized.

(1) Frequency deviation

$$
K_{f}=\frac{5}{2} \times \frac{f_{Z}-f_{N}}{f_{N}} \times 100
$$

$f_{z}$ is the real-time frequency value, $f_{N}$ is the rated frequency value, $K_{f}$ is the normal value of the frequency deviation.

(2) Voltage deviation

$$
K_{u}=\frac{100}{U_{q}} \times \frac{u_{Z}-u_{N}}{u_{N}}
$$

$u_{z}$ is the real-time voltage value, $u_{N}$ is the rated voltage value, $U_{q}$ is the voltage deviation GB allowable limit, $K_{u}$ is the voltage deviation normalized value.

(3) Voltage fluctuation and flicker

$$
K_{p}=\frac{p s t_{Z}}{p s t_{q}}
$$


$P s t_{z}$ is the real-time flicker value, $p s t_{q}$ is the voltage flicker GB allowable limit, $K_{p}$ is the flicker deviation normalized value.

(4) Harmonic distortion rate

$$
K_{A}=\frac{A}{2}
$$

$A$ is the real-time harmonic value, $K_{A}$ is the harmonic distortion rate normalized value.

(5) Odd harmonic voltage content

$$
K_{H a}=\frac{100}{H_{q}} \times \frac{\sum_{1}^{n}\left(H_{a}\right)^{2}}{\sum_{1}^{n}\left(H_{1}\right)^{2}}
$$

$H_{a}$ is the first harmonic value, $\mathrm{H}_{1}$ is the fundamental harmonic value, $H_{q}$ is the odd harmonics national standard allowable limit, $K_{H a}$ is the frequency deviation normalization value.

(6) Voltage three-phase imbalance

$$
K_{u c}=\frac{u_{c}}{2}
$$

$u_{c}$ is the voltage three-phase component, $K_{u c}$ is the voltage three-phase imbalance normalized value.

After normalization, the indicators are kept at the same level, roughly 1 as the limit, the closer to 0 indicators of the better performance. Excellent indicators in the evaluation of power quality indicators cannot play a corresponding role, and will require the need for composite single index number too much, causing interference to the calculation, should be excluded. Such as odd harmonic voltage from the third harmonic to twenty-fifth harmonic, a total of more than a dozen indicators, if all are evaluated, the index is too large and meaningless, so from the odd harmonic, And for the rest of the indicators, the normalized indicator with a target value less than 0.1 should be excluded and the remaining indicators should be retained.

It should be noted that short-term flicker indicators should not be part of a comprehensive indicator. As shown in Figure 2, short-term flicker assessment results in the vast majority of time is quite good, but the occasional jump, which is different from the other indicators of change, if and other indicators together, it will affect the comprehensive Index value, but also cannot observe the original indicators appear in the transition time period.

\section{Empowerment Algorithm}

After selecting the appropriate metric, you should calculate the weight. In this paper, subjective analytic hierarchy process and objective entropy weight method combined with the subjective and objective complex weights.

Analytic Hierarchy Process (AHP) as a way to determine the subjective weight, divided into four steps: the establishment of the hierarchical hierarchical structure of the problem, determine the comparison matrix, the calculation of weight [10]. 
Hierarchical hierarchical structures can usually be divided into target layer, criterion layer and scheme layer.

The comparison judgment matrix represents the comparison of the relative importance between the present level and its associated units for the previous hierarchy, and the scale of the importance is shown in the following Table 1 and

\section{Table 2.}

Each column of the judgment matrix is normalized:

$$
\alpha_{i j}=\frac{A_{i j}}{\sum_{i=1}^{n} A_{i j}}
$$

The normalized judgment matrix is added in rows:

$$
\alpha_{i}=\sum_{j=1}^{n} \alpha_{i j}
$$

Make Ai normalized, seeking weight:

$$
\omega_{i}=\frac{\alpha_{i}}{\sum_{i=1}^{n} \alpha_{i}}
$$

Let the weight values be sorted by weight vectors:

$$
\omega=\left(\omega_{1}, \omega_{2}, \ldots, \omega_{n}\right)^{T}
$$

Entropy method is a kind of typical objective weighting method. Many objects can be evaluated by multiple indexes. According to the weight obtained by entropy method, a certain value is often large (more than 0.3 , sometimes even up to 0.6 phenomenon, which is seriously inconsistent with the importance of indicators. Although the importance of the indicators are not the same, but there

\begin{tabular}{|c|c|}
\hline Scaling & meaning \\
\hline 1 & Two factors compared to the same importance \\
\hline 3 & Two factors, one factor is slightly more important than the other \\
\hline 5 & Two factors compared to one factor are more important than the other \\
\hline 7 & Two factors compared to one factor are more important than the other \\
\hline 9 & Two factors compared to one factor are the most important \\
\hline $2,4,6,8$ & The median of the two adjacent judgments \\
\hline reciprocal & Factor $i$ and $j$ compared to $b_{i p}$ then the factor $j$ and $i$ compared $b_{j i}=1 / b_{i j}$ \\
\hline
\end{tabular}
should not be a large indicator of the weight of the situation, or by this indicator can reflect the pros and cons of the object, Without regard to other indicators

Table 1. Matrix element scale table.

Table 2. Judgment matrix.

\begin{tabular}{cccc}
\hline Index weight & $\mathrm{A} 1$ & $\mathrm{~A} 2$ & $\mathrm{~A} 3$ \\
\hline $\mathrm{A} 1$ & 1 & $\mathrm{q}$ & $\mathrm{w}$ \\
$\mathrm{A} 2$ & $1 / \mathrm{q}$ & 1 & $\mathrm{z}$ \\
$\mathrm{A} 3$ & $1 / \mathrm{w}$ & $1 / \mathrm{z}$ & 1 \\
\hline
\end{tabular}


of the patent for the traditional entropy method to improve the objective weight calculation. The first is to establish the model, with $\mathrm{m}$ evaluation objects recorded as $M=\left(M_{1}, M_{2}, \ldots, M_{m}\right)$, Suppose there are $\mathrm{n}$ evaluation indicators recorded as $D\left(D_{1}, D_{2}, \ldots, D_{n}\right)$, The value of the evaluation object $\mathrm{M}_{\mathrm{i}}$ to the index $\mathrm{D}_{\mathrm{j}}$ is recorded as $X_{i j}(i=1,2, \ldots, m ; j=1,2, \ldots, n)$, The initial information matrix can be obtained:

$$
X=\left[\begin{array}{ccc}
X_{11} & \cdots & X_{1 n} \\
\vdots & \ddots & \vdots \\
X_{m 1} & \cdots & X_{m n}
\end{array}\right]
$$

where $X_{i j}$ is the value of the $\mathrm{i}$-th evaluated object under the jth index. The original matrix is dimensionless:

$$
v_{i j}=\frac{\max \left(x_{j}\right)-x_{i j}}{\max \left(x_{j}\right)-\min \left(x_{j}\right)}
$$

In the case of the $\mathrm{jth}$ index, the proportion of the $\mathrm{i}$-th evaluation object is $\mathrm{p}_{\mathrm{ij}}$ :

$$
p_{i j}=\frac{v_{i j}}{\sum_{i=1}^{m} v_{i j}}
$$

Calculate the entropy $e_{j}$ of the $j$ th index:

$$
e_{j}=(\ln m)^{-1} \times \sum_{i=1}^{m}\left(p_{i j} \times \ln p_{i j}\right)
$$

It should be noted that the greater the difference in the index value of each item to be evaluated, indicating that the greater the amount of information reflected by the indicator, the smaller the entropy. While the entropy is too large, indicating that the information provided by the indicator is very small, you can give it appropriate to remove it.

The difference coefficient $h_{j}$ of the jth index is:

$$
h_{j}=1-e_{j}
$$

Calculate the entropy weight $\omega_{j}$ of the jth index:

$$
\omega_{j}=\frac{h_{j}}{\sum_{j=1}^{n} h_{j}}
$$

The traditional entropy method is used as the objective weight directly after calculating the entropy weight according to the above process, but the modified entropy method needs to correct the entropy. Let the maximum entropy weight obtained by the above formula be $\omega_{j}$, and when $\omega_{j}>0.3$, it can be set to 0.3 , that is, the modified entropy weight $\omega_{i}^{\prime}=0.3$, the excess part $\left(\omega_{j}-0.3\right)$ is scaled by the following function To the remaining $(\mathrm{m}-1)$ indicators.

$$
\begin{gathered}
\omega_{i}^{\prime}=\omega_{i}+\frac{\omega_{i}}{\sum_{k=1}^{m} \omega_{k} \times\left(\omega_{j}-0.3\right)} \\
(i \neq j, i=1,2, \ldots, m)
\end{gathered}
$$

The entropy weight of each index is obtained

$$
W^{\prime}=\left(\omega_{1}^{\prime}, \omega_{2}^{\prime}, \ldots, \omega_{m}^{\prime}\right)
$$


If there is a modified entropy weight $\omega_{k}^{\prime}>0.3$ for an indicator in $W^{\prime}$, it can be reordered to 0.3 , and the weight $\left(\omega_{k}^{\prime}-0.3\right.$ ) of the redundant part is assigned to the rest $(m-2)$ indicators, and then again $(m-2)$ indicators of the revised entropy.

After obtaining the subjective and objective weight value, it will be merged into subjective and objective compound weight:

$$
\omega_{i}=\frac{1}{n} \sum_{1}^{n} u_{i} v_{i}=\left(\omega_{1}, \omega_{2}, \omega_{3}, \ldots, \omega_{n}\right)
$$

\section{Improved Radar Map Method}

Radar graph method due to the different order of the indicators caused by the evaluation results are not unique, the fundamental reason is that when drawing the radar map, the order of linear connection points on the axis of the formation of polygons [11] [12]. When constructing the evaluation function, the polygon area and the circumference are not equal due to the different indexes, and the evaluation results are inconsistent. Therefore, this paper uses the arc instead of the triangular area, and constructs the evaluation function according to the drawn radar map. Transform the composite weights obtained above into the weight angles of the improved radar graphs $\theta_{i}=2 \pi \omega_{i}$.

The center of the circle as a starting point, the horizontal right to make a ray as the first indicator of the reference axis, in which to take $\mathrm{p}_{1}$ length, with $\mathrm{n}$ indicators, then $\theta_{i}$ as the center angle for the fan radius counterclockwise for a fan, then represents the representative area of the first indicator. And then in order to as a fan radius, as the center of the center of the counterclockwise direction to make each indicator of the representative area, Six indicators of the improved radar map shown in Figure 1.

Can be calculated fan area is

$$
\mathrm{S}=\left(\sum_{1}^{n} \theta_{i} p_{i}^{2}\right)
$$

The normalized value of the composite index can be regarded as normalizing the sector area

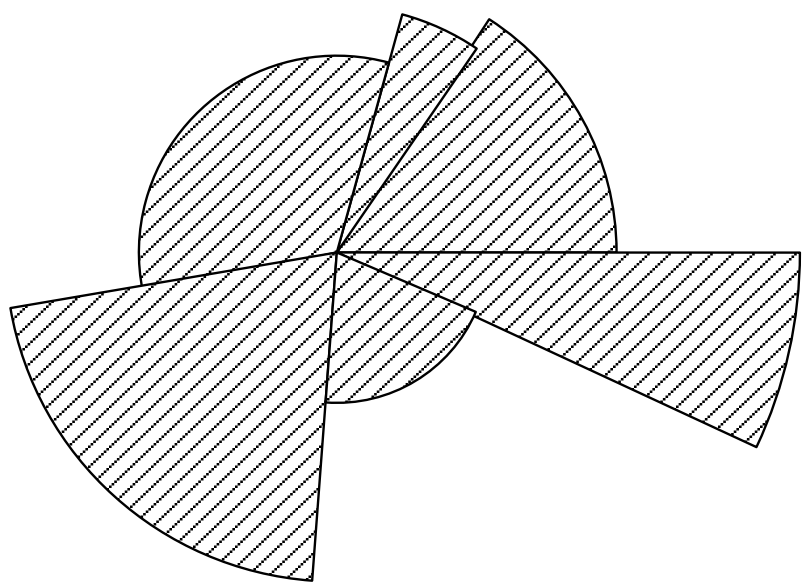

Figure 1. Example of six indicators radar. 


$$
\mathrm{Z}=\left(\sum_{1}^{n} \frac{\theta_{i} p_{i}^{2}}{2 \pi}\right)
$$

$p_{i}$ is the normalized value for the $\mathrm{i}$-th item.

After the normalized weight of the comprehensive index is determined, the fuzzy evaluation method is used to classify the obtained index.

It is very important to use fuzzy mathematics to deal with the problem of power quality evaluation and the selection and establishment of fuzzy model. The validity of the membership function directly affects the credibility of the final judgment [13]. The quality evaluation can be described by the five-level fuzzy evaluation set $\mathrm{V}$ \{excellent, good, medium, qualified, unqualified\}, and the evaluation criteria of the comprehensive index are shown in Table 3.

The establishment of a single factor evaluation matrix for each sub-index of power quality is the most critical step in evaluating the quality of power. In order to establish the univariate evaluation method of the index to be evaluated, the membership degree of the subordinate index of a specific power quality should be determined on the basis of establishing the membership function density function. Since the index is ambiguous relative to the two quality levels, the membership function between the two levels can be quantified for the five quality levels of the division, where $Z_{1}$ and $Z_{2}$ are determined by the actual The situation is determined, $\mathrm{X}$ is the index limit.

The index corresponds to the membership function for the excellent quality level

$$
\mu(Z)=\left\{\begin{array}{lc}
1, & 0 \leq Z \leq Z_{1}+C \\
\frac{1}{2}-\frac{1}{2} \sin \varphi, & Z_{1}+C<Z<Z_{2}+C \\
0, & Z \geq Z_{2}+C
\end{array}\right.
$$

In this function: $\varphi=\frac{\pi}{Z_{2}-Z_{1}}\left(Z-\frac{Z_{2}+Z_{1}}{2}\right) ; C$ is a constant, can be taken as $\frac{1}{8} X$

Indicators correspond to the membership function of good, medium and qualified quality

$$
\mu(Z)=\left\{\begin{array}{lc}
0, & Z \leq-Z_{2}+(n k+C) \\
\frac{1}{2}+\frac{1}{2} \sin \varphi, & -Z_{2}+(n k+C)<Z<-Z_{1}+(n k+C) \\
\frac{1}{2}-\frac{1}{2} \sin \varphi, & -Z_{1}+(n k+C)<Z<Z_{2}+(n k+C) \\
0, & Z \geq Z_{2}+(n k+C)
\end{array}\right.
$$

Table 3. Comprehensive index rating table.

\begin{tabular}{cccccc}
\hline Comprehensive index & excellent & good & medium & qualified & unqualified \\
\hline$Z$ & $0-0.1$ & $0.1-0.3$ & $0.3-0.6$ & $0.6-1$ & $>1$ \\
\hline
\end{tabular}


In this function: the value of parameter $\mathrm{K}$ is determined by the national standard limit, preferably $\frac{1}{4} X ; \mathrm{n}=1,2,3$.

The index corresponds to the membership function for the unqualified quality level

$$
\mu(Z)=\left\{\begin{array}{lc}
1, & Z \geq-Z_{1}+(n k+C) \\
\frac{1}{2}+\frac{1}{2} \sin \varphi, & -Z_{2}+n k+C<Z<-Z_{1}+(n k+C) \\
0, & Z \leq-Z_{2}+(n k+C)
\end{array}\right.
$$

In this function: $\mathrm{n}=4$.

The above model can be used to describe the relationship between the two quality levels, rather than the membership of the overall qualified range, by describing the membership of the individual quality evaluation index relative to each quality level.

\section{Case Study}

In this paper, three substations in Shanghai are selected to analyze the energy quality indexes of 2014, and the evaluation indexes and corresponding statistical data are shown in Table 4.

Through the consistency test, we can draw a reasonable index weight. In this paper, we select the priority judgment, frequency deviation > odd harmonics $>$ harmonic distortion $>$ voltage deviation $>$ imbalance in the evaluation of power quality in [14]. The subjective weight is calculated by analytic hierarchy process and then entropy The objective weight of the three sites is obtained, and the weight of the subjective and objective weights of the three sites is obtained. Taking $\mathrm{S} 1$ as an example, the weight values of each index are $=(0.17,0.14,0.17,0.19$, 0.22 ), They represent the odd harmonic voltage content, imbalance, frequency deviation, harmonic distortion rate, voltage deviation, S1 site drawing radar shown in Figure 2.

After the radar map processing of the composite index into the membership function, the resulting three site membership values were:

$$
\begin{aligned}
& \text { S1 }(0.211,0.462,0.156,0.098,0.046) \\
& \text { S2 }(0.018,0.145,0.236,0.347,0.514) \\
& \text { S3 }(0.425,0.301,0.157,0.115,0.023)
\end{aligned}
$$

According to the principle of maximum membership degree, the fuzzy evaluation results of three sites are as follows: S1 is good, S2 is passed and S3 is excellent. The results of this method are shown in Table 5 .

Table 4. Sample data sets to be evaluated.

\begin{tabular}{cccccc}
\hline Substation & $\begin{array}{c}\text { Voltage } \\
\text { deviation }\end{array}$ & $\begin{array}{c}\text { Harmonic } \\
\text { distortion rate }\end{array}$ & Unbalance & $\begin{array}{c}\text { Frequency } \\
\text { deviation }\end{array}$ & $\begin{array}{c}\text { Odd } \\
\text { harmonic }\end{array}$ \\
\hline S1 & 4.37 & 0.62 & 0.24 & 0.06 & 1.03 \\
S2 & 6.39 & 3.06 & 0.13 & 0.07 & 1.24 \\
S3 & 4.77 & 1.79 & 0.30 & 0.16 & 0.35 \\
\hline
\end{tabular}




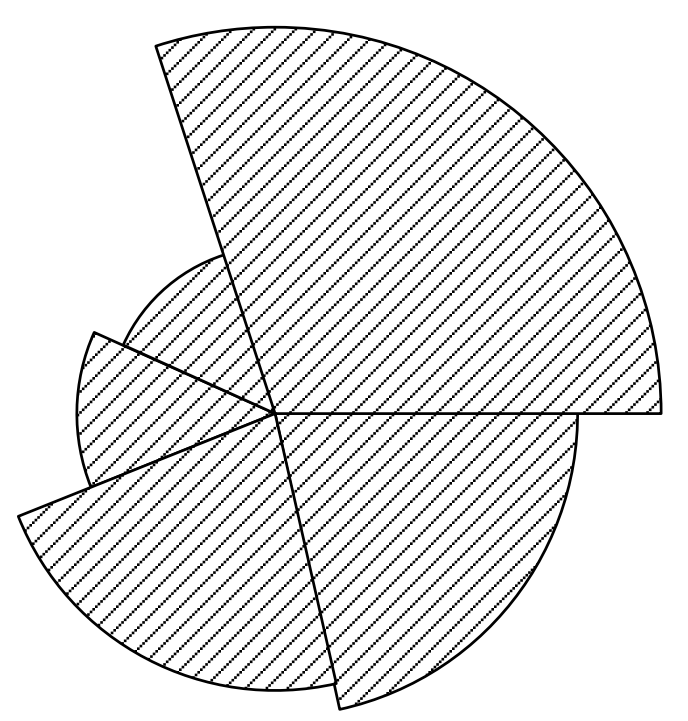

Figure 2. S1 site measurement data from the radar map.

Table 5. Comparison of the results of the comprehensive evaluation of power quality.

\begin{tabular}{ccccc}
\hline Substation & $\begin{array}{c}\text { Gray correlation } \\
\text { method }\end{array}$ & $\begin{array}{c}\text { Fuzzy } \\
\text { Mathematics }\end{array}$ & $\begin{array}{c}\text { Improved radar } \\
\text { map method }\end{array}$ & $\begin{array}{c}\text { The method of } \\
\text { this article }\end{array}$ \\
\hline S1 & excellent & good & good & good \\
S2 & Unqualified & qualified & qualified & Unqualified \\
S3 & excellent & excellent & excellent & excellent \\
\hline
\end{tabular}

It can be seen from Table 5 that the method is in good agreement with the results of other evaluation methods. It can be seen from Table 4 that the harmonic distortion rate of the substation S2 is excessive, so the power quality should be unqualified, and only the gray correlation method is correctly evaluated with the method, It can be seen that by selecting a reasonable classification interval, this method can effectively classify the power quality and prove the effectiveness of the method.

\section{Conclusion}

In this paper, the entropy weight method is used to effectively restrict the excessive weight. Appropriate indicators are selected by means of the normalization of individual indicators to ensure the accuracy of the assessment results. Combined with the radar method to deal with the comprehensive index, and then fuzzy evaluation method for classification, effectively combined with the advantages of the two methods, by comparing the results of the traditional algorithms to prove that the evaluation of this method has been greatly improved, The assessment method is reasonable and feasible.

\section{Acknowledgements}

The work was supported by Science Project of State Grid Corporation of China (520940150010). 


\section{References}

[1] Salarvand, A., Mirzaeian, B. and Moallem, M. (2010) Obtaining a Quantitative Index for Power Quality Evaluation in Competitive Electricity Market. IET Generation, Transmission and Distribution, 4, 10-823. https://doi.org/10.1049/iet-gtd.2009.0479

[2] Mamo, X. and Javerzac, J. (2001) Power Quality Indicators. 2001 IEEE Porto Power Tech Proceedings, 1-4.

[3] GB/T 12325-2008. Powerquality -Deviation of Supply Voltage.

[4] GB/T 15945-2008. Power Quality -Frequency Deviation for Power System.

[5] GB/T 15543-2008. Powerquality -Three Phase Voltage Unbalance.

[6] GB/T 14549-1993.Qualityof Electrical Energy Supply -Harmoninc in Public Supply Network.

[7] Farghal, S.A., Kandil, M.S. and Elmitwally, A. (2002) Quantifying Electric Power Quality via Fuzzy Modeling and Analytic Hierarchy. IEE Proceedings: Generation, Transmission and Distribution, 149, 44-49. https://doi.org/10.1049/ip-gtd:20020006

[8] Li, L.J., Yao, J.G., Long, L.B., et al. (2007) Application of Combination Weighing Method in Fuzzy Synthetic Evaluation of Power Quality. (2007) Automation of Electric Power Systems, 31, 56-60.

[9] Zhang, M., Lin, T., Cao, J., et al. (2008) A Comparative Study on two Methods for Synthetic Evaluation of Power Quality. Automation of Electric Power Systems, 32, 37-41.

[10] Yang, J.H., Ouyang, S., Shi, Y.L., et al. (2014) Combined Membership Function and Its Application on Fuzzy Evaluation of Power Quality. Advanced Technology of Electrical Engineering and Energy, 33, 63-69.

[11] Wang, Y.Y. (2007) The Existing Problems and Improved Measures in Synthetic Evaluation Using Radar Chart. Statistical Education, 14, 18-20.

[12] Li, G.D., Li, G.Y., Yang, X.D., et al. (2010) A Comprehensive Power Quality Evaluation Model Based on Radar Chart Method. Automation of Electric Power Systems.

[13] Liu, H.L., Liu, A.X., Zhang, B., et al. (2008) A Fuzzy Comprehensive Evaluation Method of Maintenance Quality Based on Improved Radar Chart. ISECS International Colloquium on Computing, Communication, Control and Management, 638-642.

[14] Li, Y.J. and Wang, Y.L. (2012) Application of Improved Radar Chart in Compressive Evaluation of Power Transmission and Transformation Project. North China Electric Power University, Beijing. 
Submit or recommend next manuscript to SCIRP and we will provide best service for you:

Accepting pre-submission inquiries through Email, Facebook, LinkedIn, Twitter, etc. A wide selection of journals (inclusive of 9 subjects, more than 200 journals)

Providing 24-hour high-quality service

User-friendly online submission system

Fair and swift peer-review system

Efficient typesetting and proofreading procedure

Display of the result of downloads and visits, as well as the number of cited articles Maximum dissemination of your research work

Submit your manuscript at: http://papersubmission.scirp.org/

Or contact epe@scirp.org 